\title{
Complete chloroplast genome sequence of Betula platyphylla: gene organization, RNA editing, and comparative and phylogenetic analyses
}

Sui Wang, Chuanping Yang, Xiyang Zhao, Su Chen ${ }^{*}$ and Guan-Zheng Qu*

\begin{abstract}
Background: Betula platyphylla is a common tree species in northern China that has high economic and medicinal value. Our laboratory has been devoted to genome research on B. platyphylla for approximately 10 years. As primary organelle genomes, the complete genome sequences of chloroplasts are important to study the divergence of species, RNA editing and phylogeny. In this study, we sequenced and analyzed the complete chloroplast (ср) genome sequence of B. platyphylla.

Results: The complete cp genome of B. platyphylla was 160,518 bp in length, which included a pair of inverted repeats (IRs) of 26,056 bp that separated a large single copy (LSC) region of 89,397 bp and a small single copy (SSC) region of 19,009 bp. The annotation contained a total of 129 genes, including 84 protein-coding genes, 37 tRNA genes and 8 rRNA genes. There were 3 genes using alternative initiation codons. Comparative genomics showed that the sequence of the Fagales species cp genome was relatively conserved, but there were still some high variation regions that could be used as molecular markers. The IR expansion event of B. platyphylla resulted in larger cp genomes and rps19 pseudogene formation. The simple sequence repeat (SSR) analysis showed that there were 105 SSRs in the cP genome of B. platyphylla. RNA editing sites recognition indicated that at least 80 RNA editing events occurred in the cp genome. Most of the substitutions were $C$ to $U$, while a small proportion of them were not. In particular, three editing loci on the rRNA were converted to more than two other bases that had never been reported. For synonymous conversion, most of them increased the relative synonymous codon usage (RSCU) value of the codons. The phylogenetic analysis suggested that B. platyphylla had a closer evolutionary relationship with B. pendula than B. nana.
\end{abstract}

Conclusions: In this study, we not only obtained and annotated the complete cp genome sequence of $B$. platyphylla, but we also identified new RNA editing sites and predicted the phylogenetic relationships among Fagales species. These findings will facilitate genomic, genetic engineering and phylogenetic studies of this important species.

Keywords: Betula platyphylla, White birch, Chloroplast genome, RNA editing, Phylogeny

\section{Background}

Betula platyphylla, or Asian white birch, is a broadleaved deciduous hardwood tree species that belong to the genus Betula, in the family Betulaceae. It is a pioneer tree species that can rapidly colonize open ground, especially in secondary successional sequences. It grows in

\footnotetext{
* Correspondence: chensunefu@163.com; quguanzheng@yahoo.com State Key Laboratory of Tree Genetics and Breeding, Northeast Forestry University, 26 Hexing Road, Harbin 150040, China
}

the temperate or subarctic regions of Asia, including Japan, China, Korea, and Siberia. The grayish bark of this tree is marked with long, horizontal lenticels that often separates into thin, papery plates, which is the most typical characteristic of this tree species $[1,2]$. $B$. platyphylla is often used as a wayside tree or landscape tree species because of its graceful shape. It is a valuable commercial tree species that is harvested for lumber and pulpwood for paper production [3]. Recent studies have 
indicated that birch bark contains numerous triterpenoids and has substantial medicinal value $[4,5]$.

As primary plastids found only in plant cells and eukaryotic algae, chloroplasts are semiautonomous organelles that are not only perform photosynthesis but also participate in a range of biochemical processes. Chloroplasts are believed to have arisen from an endosymbiotic event and have their own genomes, which are often abbreviated as cp or ct $[6,7]$. Since the first cp genomes were sequenced in 1986, more than 2500 complete cp genome sequences have been released in the National Center for Biotechnology Information (NCBI) organelle genome database as of March 2018 [8, 9]. The advent of next-generation-sequencing (NGS) technologies has facilitated rapid progress in the field of cp genomics [10]. In the future, due to the popularity of third-generation sequencing, longer average read lengths will make it easier to assemble cp genomes [11-13]. For most land plants, cp genomes have highly conserved structures and are circular DNA molecules that comprise two inverted repeats (IR), which separate a large and a small single copy (LSC and SSC) region. Chloroplast genome sizes vary between species, ranging from $107 \mathrm{~kb}$ (Cathaya argyrophylla) to $218 \mathrm{~kb}$ (Pelargonium $\times$ hortorum), with an average size of approximately $150 \mathrm{~kb}[14,15]$. There are approximately 120-130 genes in the cp genome, which participate primarily in photosynthesis, transcription, and translation [16]. RNA editing, which is a posttranscriptional modification phenomenon, occurs in some transcripts of these $\mathrm{cp}$ genes. Editing by insertion, deletion or switching bases, such as cytidine $(\mathrm{C})$ to uridine $(\mathrm{U})$, is an essential repair mechanism, and many mutations at the cp genome level may lead to strong deleterious phenotypes [17]. Because they have fairly stable structures, moderate evolutionary rates and uniparental inheritance in most angiosperms, the cp genomes have made significant contributions to phylogenetic studies $[16,18]$.

In this study, we aimed to determine the complete $\mathrm{cp}$ genome sequence of $B$. platyphylla and to characterize its genome structure, gene content and other characteristics. Furthermore, we recognized RNA editing sites in the whole cp genome of B. platyphylla using RNA-Seq data. We predicted their relationships through a comparative analysis with other Fagales species cp sequences within phylogenetic clades.

\section{Materials and methods}

\section{Plant materials and sequencing}

Tender leaves were collected from an adult B. platyphylla plus tree that is located on the Northeast Forestry University campus. Total genomic DNA was extracted from tender leaves using the CTAB method [19]. Three paired-end (insert sizes $=200 \mathrm{bp}, 500 \mathrm{bp}$ and $800 \mathrm{bp}$ ) and three mate-pair (insert sizes $=2 \mathrm{kbp}, 5$ $\mathrm{kbp}$ and $10 \mathrm{kbp}$ ) Illumina libraries were prepared and sequenced on the HiSeq 2000 platform (Illumina, USA) at BGI (Shenzhen,Guangdong,China).

\section{Data filtration and cp DNA sequence extraction}

To obtain high-quality and vector/adaptor-free reads, raw paired-end reads were filtered using the NGSQC Toolkit v2.3.3 (cut-off read length for $\mathrm{HQ}=70 \%$, cut-off quality score $=20$, trim reads from $5^{\prime}=3$, trim reads from $3^{\prime}=7$ ) [20]. The qualities of the clean reads were checked using FastQC (v0.11.5). To identify the cp sequences, all of the clean reads, which included sequences from both the nucleus and organelles, were mapped to the complete cp genome sequences of 2670 plant species, which were downloaded from the NCBI Organelle Genome Resources database (www.ncbi.nlm. nih.gov/genome/organelle/) using BWA (v0.7.13) [21]. Finally, we extracted cp sequences from the SAM files and obtained three files of paired-end reads.

\section{Genome assembly and annotation}

For de novo cp genome assembly, an Edena assembler (v3.131028) with default parameters was used to assemble all the paired-end sequences into contigs [22]. Next, neighboring contigs with paired-end or mate-pair support for continuity were merged into scaffolds using SSPACE (v3.0) [23]. Then, using the cp genome sequences of two other reference Fagales plants, Betula nana (KX703002.1) and Ostrya rehderiana (KT454094.1), a single cp sequence with gaps was assembled. After that, GapCloser (v1.12) was used to close most of the gaps, and Sanger sequencing was used to fill residual gaps. The complete cp genome sequence was further checked using BWA.

Except for tRNA genes, which were verified using tRNAscan-SE 2.0, the B. platyphylla $\mathrm{cp}$ genome sequence was annotated using the online Chloroplast Genome Annotation, Visualization, Analysis and GenBank Submission Tool (CpGAVAS) [24, 25]. First, AnnotateGenome was utilized to obtain the primitive annotation results in the GFF3 format. Second, we used AnnotateGene and Apollo Genome Annotation and Curation Tool (v1.11.8) to manually correct the abnormal features based on the reference database of CpGAVAS and the tRNA genes annotated by tRNAscan-SE. Last, OrganellarGenomeDRAW was used to directly generate a corrected cp circular map [26].

\section{Codon usage and alternative start codons statistics}

Codon usage was determined for all protein-coding genes (RNA sequences without editing). To examine the deviation in synonymous codon usage while avoiding the influence of the amino acid composition, the relative synonymous codon usage (RSCU) was calculated with MEGA 7 software (version 7.0.18). 
Three cp genes ( $r p s 19, p s b C$ and $n d h D)$ were annotated with the Non-ATG start codon in the B. platyphylla cp genome, we selected these genes from 30 model plant and representative plant species according to the Angiosperm Phylogeny Group (APG) IV system (Additional file 1: Table S1). Then, the sequence logos of the first $10 \mathrm{bp}$ of the three genes across the species were created using the WebLogo 3 application (http://weblogo.threeplusone. $\mathrm{com} /$ ). We also visualized the RNA-Seq mapping of these sites and aligned them with the sequence logos.

\section{Genome comparison}

The complete cp genome sequences of B. platyphylla and four other closely related species, $B$. pendula (LT855378.1), B. nana (KX703002.1), Corylus chinensis (KX814336.2) of Betulaceae and Juglans sigillata (KX424843.1) of Juglandaceae, were compared using the program mVISTA. EMBOSS Stretcher, a modification of the Needleman-Wunsch algorithm that allows larger sequences to be aligned globally, was used to align these $\mathrm{cp}$ genome sequences to obtain accurate identity and similarity.

\section{IR expansion and contraction}

Depending on the classification system for Fagales taxa, four species Betula platyphylla, Juglans regia (MF167 463.1), Morella rubra (KY476637.1) and Castanea mollissima (KY951992.1) were selected to represent the families Betulaceae, Juglandaceae, Myricaceae and Fagaceae, respectively.

\section{SSR analysis}

Simple sequence repeats (SSRs) were detected using the Perl script MISA (MIcroSAtellite identification tool) by setting the minimum number of repeats to $10,5,4,3,3$ and 3 for mono-, di-, tri-, tetra-, penta- and hexanucleotides, respectively. Meanwhile, CandiSSR was used to identify polymorphic SSRs (PolySSRs) and to automatically design primer pairs for each identified PolySSR in the three Betula species [27].

\section{Recognition of RNA editing sites}

In this study, an RNA-Seq experiment with 3 individual leaf samples was used to identify RNA editing events. The total RNA was extracted from mature foliage using an Extract kit (RP3301, BioTeke, China). The RNA-Seq library construction and sequencing were performed at Novogene Bioinformatics Technology Co., Ltd. (Beijing, China). The filtered paired-end reads obtained from an Illumina HiSeq 2000, were aligned to the B. platyphylla cp genome using HISAT2 (v2.1.0) software with strict comparison conditions. SAMtools (v1.9), bedtools (v2.25.0) and ChloroSeq were used to call and analyse precise RNA editing sites
[28]. Because SNPs or mismatches may interfere with the results, we also mapped the set of PE $100 \mathrm{bp-long} \mathrm{reads}$ that was used to assemble the B. platyphylla $\mathrm{cp}$ genome back to the cp genome sequence using bowtie2 (v2.3.4.1) software and then checked the SNPs. Finally, we designed several pairs of primers using Primer Premier 6.0 software (PREMIER Biosoft International, Canada) and amplified the target sequence by $\mathrm{PCR}$ to form genomic DNA (gDNA) and complementary DNA (cDNA). The target representative editing sites were confirmed by Sanger sequencing. The relevant primer information is summarized in Additional file 1: Table S2.

\section{Phylogenetic analysis and character evolution}

The whole cp genome sequences of 21 species of Fagales were used to build a phylogenetic tree to confirm the genetic relationship among closely related species of $B$. platyphylla. In this phylogenetic tree, Nicotiana tabacum was used as the out-group. Nucleotide sequences were aligned using MAFFT (version 7.294b). All alignments were checked and adjusted manually. The program MEGA-CC (version 7.0.26-1) was employed to find an optimal substitution model and to build a maximum likelihood (ML) phylogenetic tree. Bootstrap resampling with 500 replicates was used to evaluate the branch supports. More information is summarized in the Additional file 1: Table S3.

\section{Results}

\section{Chloroplast genome assembly}

Based on the NCBI Organelle Genome Resources database, we extracted approximately $128.8 \mathrm{Mbp}$ of pairedend reads for $\mathrm{cp}$ genome assembly. With the help of Edena, a first assembly consisting of 35 contigs was obtained (Table 1). Further scaffolding with all of the paired-end and mate-pair reads resulted in a single scaffold under the guidance of the reference sequences. After using GapCloser to close most of the gaps, only two gaps remained. Finally, with the aid of Sanger sequencing, we filled the gaps, identified both ends of the sequence and obtained a circular cp genome.

The whole cp genome of $B$. platyphylla had a length of $160,518 \mathrm{bp}$. Like most land plants, the circular cpD NA had typical quadripartite structures. An LSC region of 89,397 bp and an SSC region of 19,009 bp were separated by a pair of IR regions of $26,056 \mathrm{bp}$. The overall $\mathrm{GC}$ content of the B. platyphylla $\mathrm{cp}$ genome was $36.06 \%$, and the GC contents of the LSC and the SSC regions were 33.66 and $29.76 \%$, respectively. Because each IR region contained relatively abundant GC-rich rRNA and tRNA genes, the GC content of the IR region was $42.48 \%$, which was much higher than that of the LSC and SSC regions. 
Table 1 Statistics for the contigs

\begin{tabular}{lccccc}
\hline Number & Size $(\mathrm{bp})$ & N50 $(\mathrm{bp})$ & N90 $(\mathrm{bp})$ & Longest length (bp) & Shortest length \\
\hline 35 & 150,362 & 21,011 & 2253 & 35,505 & 139 \\
\hline
\end{tabular}

\section{Chloroplast genome annotation}

A total of 129 genes were predicted to be encoded in the B. platyphylla cp genome, including 84 protein-coding genes, 37 tRNA genes and 8 rRNA genes. Among them, 95 genes were unique, and 17 genes were duplicated in the IR regions. By calculating the $\mathrm{GC}$ content of the genes, we found that it was higher in the rRNAs (54.89\%) and tRNAs (53.20\%) than in the protein-coding genes (36.93\%). The majority of 112 unigenes were single-exon genes, while 18 genes (12 protein-coding genes and 6 tRNA genes) contained 2 exons and only 4 protein-coding genes contained 3 exons. All of the genome and annotation information is shown in Fig. 1.

Among the $B$. platyphylla cp genes, several were special. The rps12 gene was a trans-spliced gene that consisted of 3 exons that code for the homologous ribosomal protein S12. C-terminal exons 2 and 3 of rps 12 were located in each IR region, but exon 1 was located in the LSC, approximately $28 \mathrm{kbp}$ downstream of the nearest copy of exon 2, which was located in one of the IR regions and 61 kbp away from the other copy of exon 3, which was located in the other IR region. Prediction of the B. platyphylla cp gene function was based on homology, as these genes code for a variety of proteins, mostly involved in photosynthesis and other metabolic processes. Regarding photosynthesis, a subset of the genes synthesizes the large Rubisco subunit and thylakoid proteins. In addition, other genes encode subunits of a protein complex that mediates redox reactions to recycle electrons. Table 2 shows the gene functions and groups in the B. platyphylla cp genome.

\section{Codon usage and alternative initiation codons statistics}

It is generally acknowledged that codon biases reflect a balance between mutational biases and natural selection for translational optimization. We further analyzed the codon usage frequency and RSCU value in the B. platyphylla cp genome. It was not clear whether RNA editing occurred in these areas because there were some regions covered with no reads in our experiment, and the editing rates were not $100 \%$. Here, we used RNA sequences without editing to compute the codon usage and RCSU values. We estimated that this would not have a large impact on the results. There were 84 protein-coding genes in the B. platyphylla cp genome, including 26,298 codons in total. Among the codons, the three amino acids present in the highest proportions were leucine (10.49\%), isoleucine (8.97\%) and serine (7.49\%). Excluding the stop codons, cysteine $(1.16 \%)$ was the least abundant amino acid (Additional file 1: Table S4, Figure $\mathrm{S} 1)$. Codon usage was biased towards $\mathrm{A}$ and $\mathrm{U}$ at the thirdcodon position, which is similar to the trend that was observed in a majority of angiosperm cp genomes [29].

Unlike ordinary genes that use ATG as their initiation codons, several cp genes use other codons as exceptions. In the B. platyphylla cp genome, three genes were annotated with Non-ATG start codon: GTG was used by rps 19 and $p s b C$ and ACG was used by $n d h D$. These three genes are involved in translation, photosynthesis and respiration, respectively. As shown in Fig. 2a, these selected gene sites were relatively conserved across species. GTG was the dominant initiation codon in rps19, but not in $p s b C$, and about half of the species took ACG as the start codon in $n d h D$. Figure $2 \mathrm{~b}$ shows that, at the transcriptional level, the initiation codon of rps19 and the $p s b C$ transcripts of $B$. platyphylla did not change significantly. However, editing ACG to AUG at the $n d h D$ start codon was obvious and made its start codon go back to normal.

\section{Comparison of chloroplast genome sequences with those of other species}

To investigate the similarities and differences of the cp genome sequences between B. platyphylla and other species of Fagales, a global alignment program was used to align these sequences. The result was plotted using the mVISTA tools with $B$. platyphylla as a reference (Fig. 3). Overall, these closely related species had little difference in cp genome size, ranging from $160,320 \mathrm{bp}$ to $161,148 \mathrm{bp}$. The global patterns of sequence similarities among these sequences were very high, especially among the Betulaceae species, with over 99\% identity. As shown in Fig. 3, the structures of these $\mathrm{cp}$ genomes were conserved, and neither translocations nor inversions were detected in the sequences. As expected, coding regions were revealed to be more conserved than noncoding regions. More concretely, most high polymorphic regions were located in the intergenic regions (such as trnR-TCT-atpA, trnE-TTC-trnT-GGT, psbEpetL, rpl32-trnL-TAG), but the $y c f 1$ gene had higher variability regions. These regions may be undergoing more rapid nucleotide substitution at the species level, which indicates the potential application of molecular markers for phylogenetic analyses and plant identification in Fagales.

\section{IR expansion and contraction}

IR expansions and contractions are common in cp genomes, which results in the variation in $\mathrm{cp}$ genome size. 


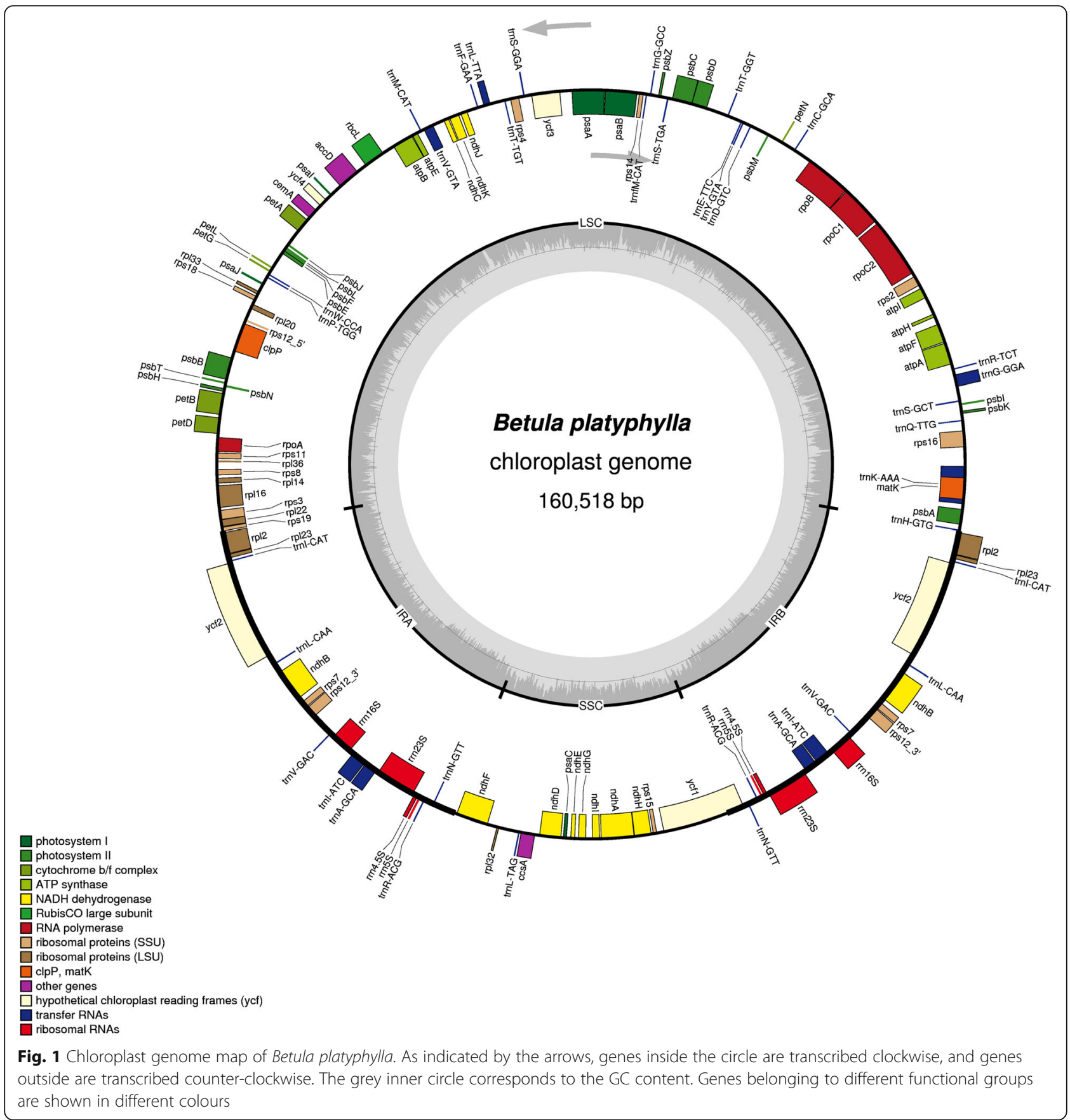

The differences in IRs may also reflect phylogenetic history. Here, we selected four representative species of the four families in Fagales and compared their sizes and the junctions of their LSC, SSC and IR regions. Although the lengths of the IR regions, ranging from $25,701 \mathrm{bp}$ to $26,056 \mathrm{bp}$, varied little among the four species, some differences in the IR expansions and contractions were observed. As shown in Fig. 4, B. platyphylla did not show the longest total cp genome length among the four species, but its IR regions were the longest. The rps19 genes of J. regia, M. rubra and C. mollissima were located in the LSC region, but the IRb region was expanded to include the rps19 gene in B. platyphylla. Although the $r p l 2$ genes of the four species were all located completely within the IRb regions, the gene in $B$. platyphylla was at the farthest from the left boundary of the IR region. The $\psi y c f 1$ pseudogene was present in all four genomes, except in J. regia, in which it extends into 
Table 2 Group of genes within the B. platyphylla chloroplast genome

\begin{tabular}{|c|c|}
\hline Group of genes & Gene names \\
\hline Photosystem I & psaA, psab, psaC, psal, psaJ \\
\hline Photosystem ॥ & $p s b A, p s b B, p s b C, p s b D, p s b E, p s b F, p s b H, p s b l, p s b J, p s b K, p s b L, p s b M, p s b N, p s b T, p s b Z$ \\
\hline Cytochrome b/f complex & petA, petB, petD, petG, petL, petN \\
\hline ATP synthase & $\operatorname{atp} A, \operatorname{atp} B, \operatorname{atp} E, \operatorname{atp} F, \operatorname{atpH}, \operatorname{atpl}$ \\
\hline NADP dehydrogenase & $n d h A, n d h B, n d h C, n d h D, n d h E, n d h F, n d h G, n d h H, n d h l, n d h J, n d h K$ \\
\hline RubisCO large subunit & $r b c L$ \\
\hline RNA polymerase & rpoA, rpoB, rpoC1, rpoC2 \\
\hline Ribosomal proteins (SSU) & rps2, rps3, rps4, rps7, rps8, rps11, rps12, rps 14, rps15, rps16, rps18, rps19 \\
\hline Ribosomal proteins (LSU) & rpl2, rp/14, rp/16, rpl20, rpl22, rp/32, rp/33, rp/36 \\
\hline Hypothetical chloroplast reading frames(ycf) & $y c f 1, y c f 2, y c f 3, y c f 4$ \\
\hline Other genes & $a c c D, c c s A, c e m A, c l p P$, matk \\
\hline Ribosomal RNAs & $r r n 4.5 S, r r n 5 S, r r n 16 S, r r n 23 S$ \\
\hline Transfer RNAs & 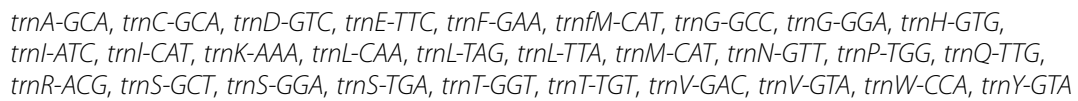 \\
\hline
\end{tabular}

the SSC region by several bases; the others were all located in the IRb regions. The distance between the $\psi y c f 1$ pseudogene and the $n d h F$ gene decreased from $B$. platyphylla to C. mollissima. The IRa region extended into the $y c f 1$ gene in all these genomes, and the longest overlap between the IRa and $y c f 1$ genes was also observed in B. platyphylla. Of particular interest, the urps19 pseudogene only existed in the B. platyphylla genome, which is located along the right boundary of the IRa region. The trnH-GTG genes were all located in the LSC region, at $7-47 \mathrm{bp}$ apart from the IRa-LSC boundary. In summary, we found that the IR regions of the $B$. platyphylla cp genome were slightly expanded compared with that of the other three species.

\section{SSR analysis}

Recently, an increasing number of theoretical reasons and well-documented examples show that the repetitive structure of genomic DNA is essential. It not only plays a major architectonic role in higher-order physical structuring but is also very useful in genome evolution and rearrangement [30, 31]. Here, we only focused on microsatellite sequences. A total of 105 SSRs were detected in the B. platyphylla cp genome (Additional file 1: Table S5). Among these SSRs, there were 53, 19, 15, 13 and 5 for mono-, di-, tri-, tetra- and penta- nucleotide repeats, respectively. No hexanucleotide repeats were found. A majority of the mononucleotides $(98.1 \%)$ were composed of $\mathrm{A} / \mathrm{T}$, and most of the dinucleotides (84.2\%) were AT

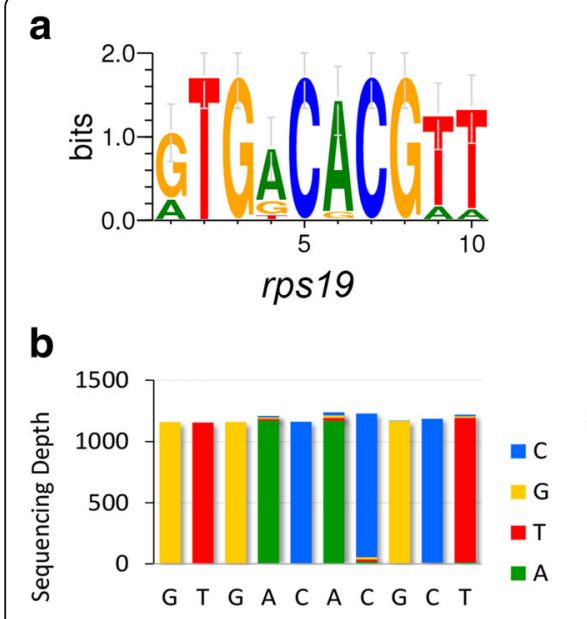

rps19
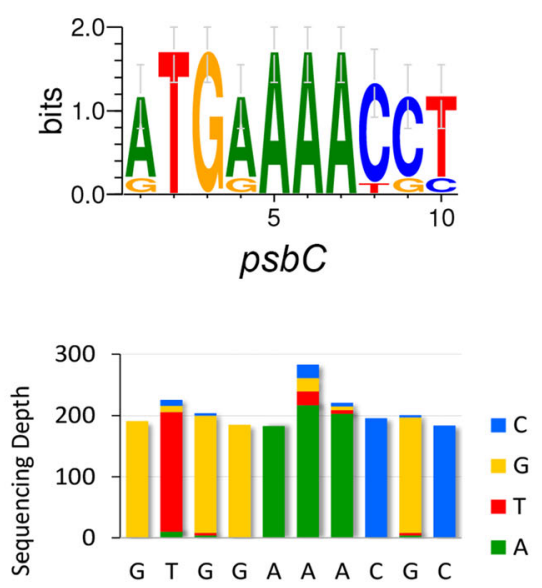

$p s b C$
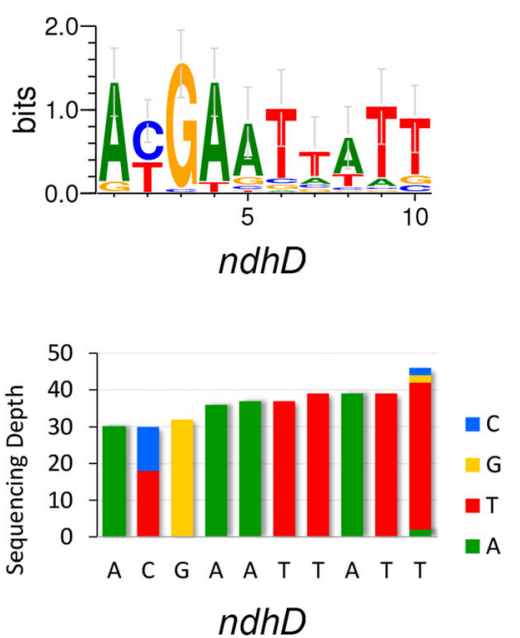

Fig. 2 Sequence logo and RNA-Seq mapping of the three genes. a: Sequence logo of the first $10 \mathrm{bp}$ of the three genes across the species. $\mathbf{b}$ : RNA-Seq mapping of the first $10 \mathrm{bp}$ of the three genes in B. platyphylla 


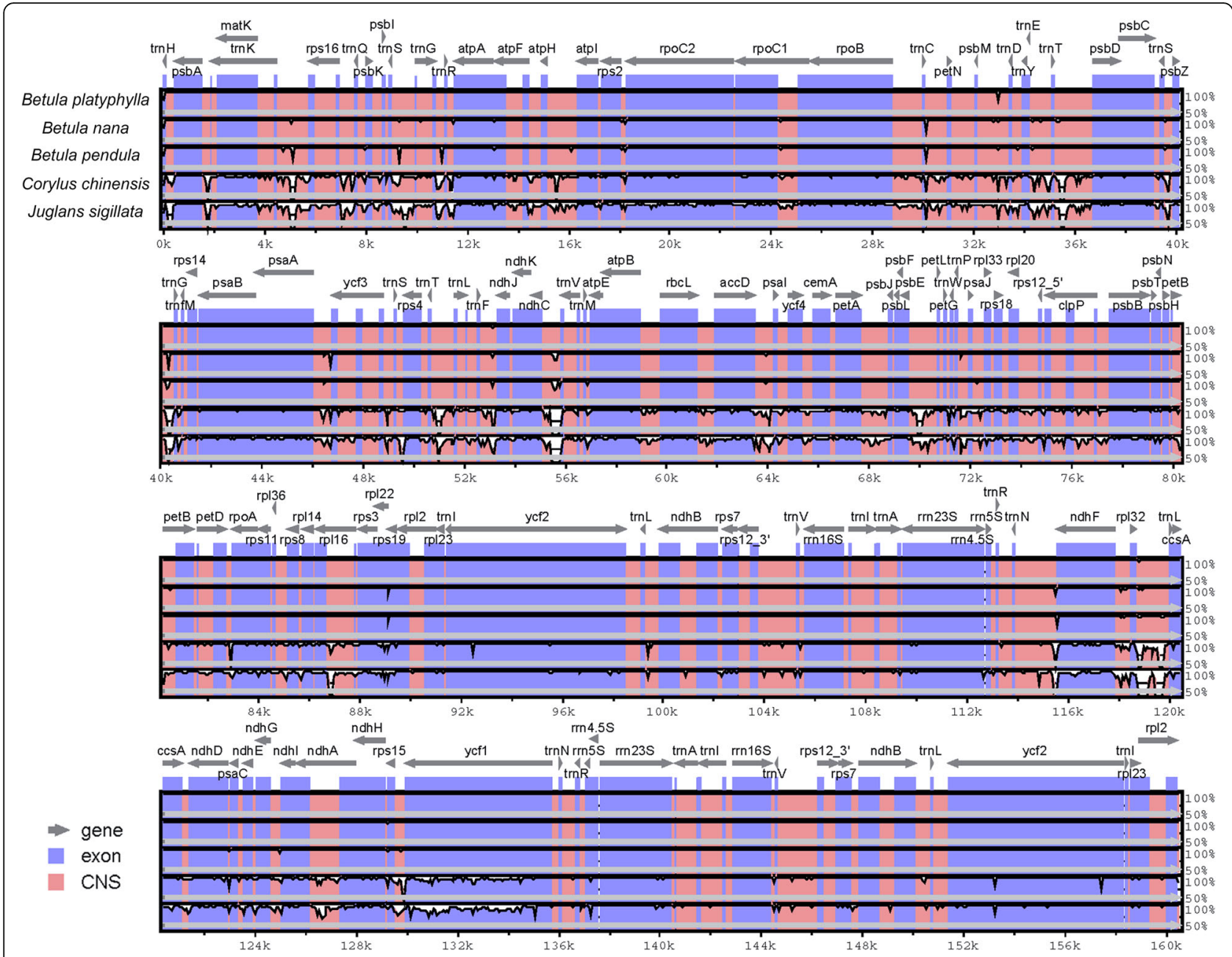

Fig. 3 Sequence alignment of 5 chloroplast genomes in Fagales using the mVISTA program with B. platyphylla as a reference. Grey arrows above the alignment indicate the transcriptional directions of genes. Genome regions are color-coded as exon and conserved non-coding sequences (CNS). A cut-off of $50 \%$ identity was used for the plots. The Y-axis indicates the percent identity between 50 and 100\%

(Additional file 1: Future S4). Because repeat sequences may be useful in developing lineage-specific markers, CandiSSR was used to identify candidate polymorphic SSRs among the cp genomes. Nevertheless, we only found one possible SSR locus among the three Betula species (Additional file 1: Table S6).

\section{Recognition of RNA editing sites}

To exhaustively analyze the B. platyphylla cp genome RNA editome, high-quality reads are necessary. After data cleaning, approximately $11.7 \mathrm{Gbp}$ clean reads were obtained. The read alignment rates of the three biological repetitions to the reference $B$. platyphylla $\mathrm{cp}$ genome were only $1.23,0.67$ and $0.67 \%$. However, a $95.3 \%$ region of the cp genome was covered with reads, and the average sequencing depth was over 650x. Furthermore, the distribution of reads was not uniform; the region in which tRNA and rRNA genes clustered in the IR had extremely high sequencing depth. The genome and gene body coverage maps are shown in the Additional file 1: Figures S2 and S3. Finally, we identified 80 RNA editing events in the whole $B$. platyphylla cp genome, of which 73 and 7 were located in gene and intergenic regions, respectively (Table 3). Among all of the edited genes, most of them were protein-coding genes, while 3 of them were rRNA genes. We did not discover any editing events that occurred in the tRNA transcripts. In protein-coding transcripts, editing events mainly occurred in the codon region, with only 1 in the 5 ' UTR and 4 in introns. The largest number of editing sites was recognized in the $n d h B$ gene (10 editing sites). A majority of changes in protein-coding transcripts converted $\mathrm{C}$ to $\mathrm{U}$ (5'-3' direction of transcription), along with $\mathrm{G}$ to $\mathrm{A}$ and $\mathrm{C}$ to $\mathrm{G}$ in $r p o C 2$, A to $\mathrm{G}$ in $y c f 2$ and $\mathrm{G}$ to $\mathrm{A}$ in $n d h F$. Among the edited codons, there were $9(13.8 \%)$ changes at the first position, $49(75.4 \%)$ 


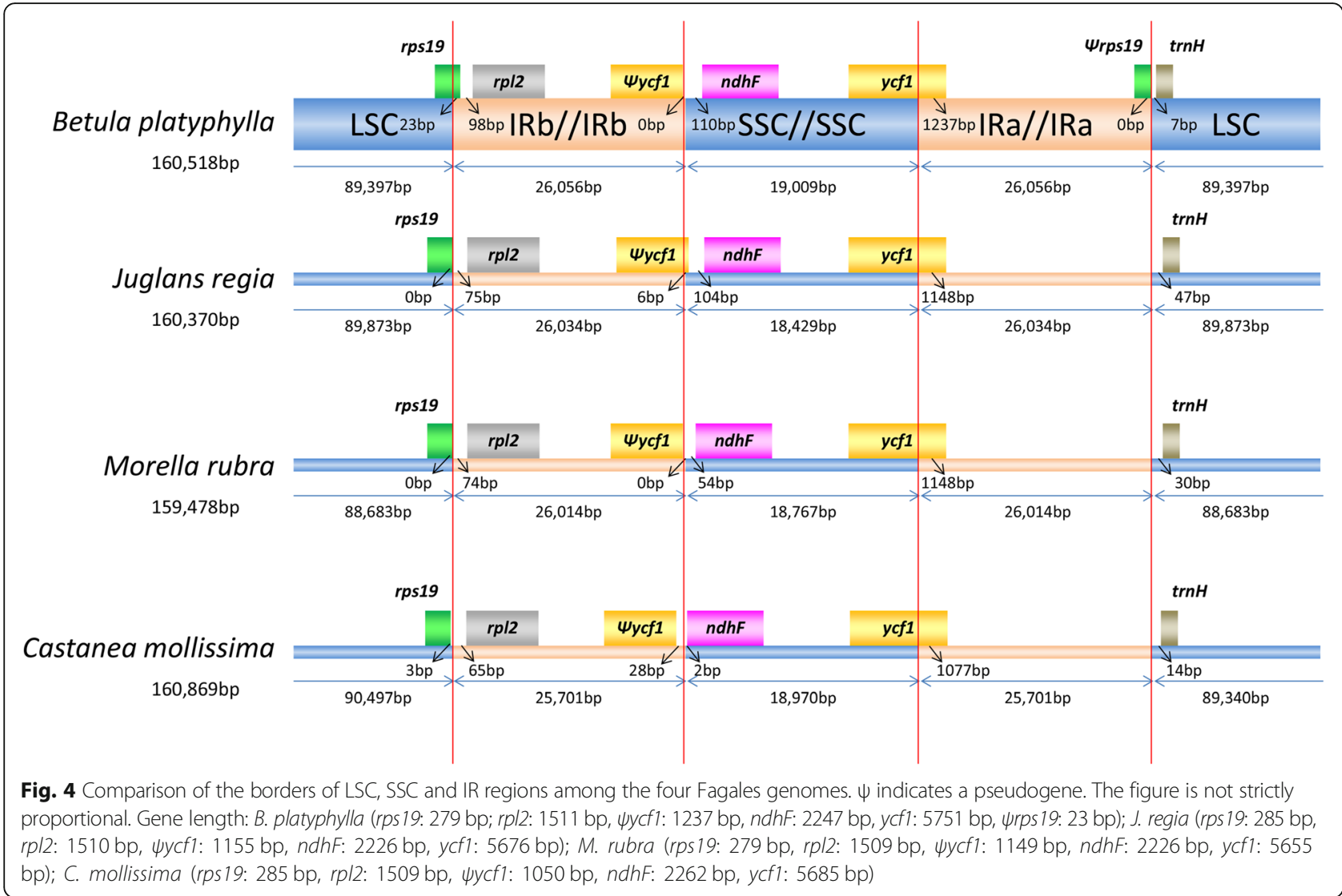

at the second position and $7(10.8 \%)$ at the third position. Most editing events (58 sites, 89.2\%) lead to amino acid changes and editing in 7 loci $(10.8 \%)$ did not alter amino acids (including stop codons). Among the 58 amino acid change sites, the largest proportion were Ser to Leu ( 25 sites, $43.1 \%$ ), followed by Pro to Leu (12 sites, 20.7\%) and Ser to Phe (9 sites, 15.5\%). After comparison with the codon usage frequency and RSCU value at the editing sites, except for stop codon conversion, we found that all 6 other amino acid synonymous conversions increased the RSCU value of the codons, whereas the other nonsynonymous conversions did not show the same trend. For several editing sites located in the Intergenic region, nucleotide changes, including $\mathrm{G}$ to $\mathrm{A}, \mathrm{C}$ to $\mathrm{U}$ and $\mathrm{A}$ to $\mathrm{C}$ (genome sequence direction as a positive chain), were observed. However, the editing sites located in the rRNA were the most specific; each site may be converted to more than two other bases. We also recognized that different editing sites had different editing efficiencies, from $~ 100 \%$ greater to $\sim 10 \%$ poorer. As shown in Fig. 5 , the $\mathrm{C}$ to $\mathrm{T}$ transitions at the 1406th base of the $a c c D$ gene and at the 155 th base of the matK gene were noticeable. After careful observation of the cDNA region in Fig. 5, it is not difficult to see that there is a weak cytosine $(\mathrm{C})$ base signal under the peak of the corresponding editing site.

\section{Phylogeny inference}

To determine the position of B. platyphylla in Fagales and to further analyze the relationships within Fagales, the complete cp genome sequences of 21 Fagales were obtained from the NCBI. Due to the high similarity of protein sequences in related species, we employed the whole cp genome sequences to build the phylogenetic tree to obtain more information. Model testing showed that GTR + G+I (General Time Reversible model, Gamma Distributed with Invariant Sites) was the optimal mode for whole-genome nucleotide sequences. In the latest APG IV system, Fagales includes seven families (Nothofagaceae, Fagaceae, Myricaceae, Juglandaceae, Ticodendraceae, Betulaceae, Casuarinaceae). In our study, the selected species only came from four families. Figure 6 clearly shows that all species formed four major clades, which correspond to the four selected families. In our ML tree, Fagaceae were sister to the remaining Fagales, followed by Myricaceae, which were subsequently sister to the remaining Fagales. Between B. platyphylla, B. pendula and B. nana of the genus Betula and family Betulaceae, B. platyphylla and $B$. pendula were located in adjacent branches, which means that they may have a closer evolutionary relationship. 
Table 3 RNA editing sites and amino acid changes

\begin{tabular}{|c|c|c|c|c|c|c|c|c|c|}
\hline Gene & Subunit & Genome Position & Gene Position & Nucleotide Change & Codon Change & $\begin{array}{l}\text { Edit Position within } \\
\text { Codon }\end{array}$ & $\begin{array}{l}\text { Amino Acid } \\
\text { Change }\end{array}$ & $\begin{array}{l}\text { Sequencing } \\
\text { Depth }\end{array}$ & $\begin{array}{l}\text { Editing } \\
\text { Efficiency }\end{array}$ \\
\hline matk & exon & 2963 & 710 & $G>A$ & $U C U>U U U$ & 2 & $S>F$ & 37 & 70 \\
\hline matk & exon & 3518 & 155 & $G>A$ & $U C U>U U U$ & 2 & $S>F$ & 38 & 84 \\
\hline rps16 & exon & 5795 & 212 & $G>A$ & $U C A>U U A$ & 2 & $S>L$ & 179 & 82 \\
\hline rps16 & intron & 6389 & - & $G>A$ & - & - & - & 47 & 81 \\
\hline $\operatorname{atp} A$ & exon & 12,099 & 914 & $G>A$ & $U C A>U U A$ & 2 & $S>L$ & 120 & 98 \\
\hline $\operatorname{atpA}$ & exon & 12,222 & 791 & $G>A$ & $C C G>C U G$ & 2 & $P>L$ & 189 & 98 \\
\hline $\operatorname{atpF}$ & exon & 14,294 & 92 & $G>A$ & $C C A>C U A$ & 2 & $P>L$ & 199 & 81 \\
\hline$I G R-1$ & IGR & 16,247 & - & $G>A$ & - & - & - & 229 & 97 \\
\hline rps2 & exon & 17,781 & 248 & $G>A$ & $U C A>U \cup A$ & 2 & $S>L$ & 85 & 95 \\
\hline rpoC2 & exon & 18,698 & 3761 & $G>A$ & $U C A>U U A$ & 2 & $S>L$ & 41 & 98 \\
\hline rpoc2 & exon & 20,661 & 1798 & $G>T$ & $\mathrm{GGU}>\mathrm{AGU}$ & 1 & $G>S$ & 33 & 18 \\
\hline rpoc2 & exon & 20,763 & 1696 & $G>C$ & $\mathrm{CUC}>\mathrm{GUC}$ & 1 & $L>V$ & 34 & 12 \\
\hline rpoCl & exon & 24,185 & 488 & $G>A$ & $U C A>U U A$ & 2 & $S>L$ & 79 & 80 \\
\hline rpoCl & exon & 25,453 & 41 & $G>A$ & $U C A>U U A$ & 2 & $S>L$ & 20 & 65 \\
\hline$r p o B$ & exon & 26,307 & 2426 & $G>A$ & $U C A>U U A$ & 2 & $S>L$ & 11 & 100 \\
\hline$r p o B$ & exon & 26,733 & 2000 & $G>A$ & $U C U>U U U$ & 2 & $S>F$ & 15 & 93 \\
\hline$r p o B$ & exon & 27,555 & 1178 & $G>A$ & $U C G>U U G$ & 2 & $S>L$ & 12 & 67 \\
\hline$r p o B$ & exon & 28,167 & 566 & $G>A$ & UCG $>$ UUG & 2 & $S>L$ & 6 & 17 \\
\hline$r p o B$ & exon & 28,182 & 551 & $G>A$ & $U C A>U U A$ & 2 & $S>L$ & 9 & 44 \\
\hline$r p o B$ & exon & 28,395 & 338 & $G>A$ & $U C U>U U U$ & 2 & $S>F$ & 22 & 86 \\
\hline$I G R-2$ & IGR & 35,186 & - & $A>C$ & - & - & - & 23 & 17 \\
\hline$p s b Z$ & exon & 39,947 & 50 & $C>U$ & $U C A>U \cup A$ & 2 & $S>L$ & 176 & 26 \\
\hline rps 14 & exon & 41,250 & 149 & $G>A$ & $C C A>C U A$ & 2 & $P>L$ & 210 & 90 \\
\hline rps 14 & exon & 41,319 & 80 & $G>A$ & $\mathrm{CCC}>\mathrm{CUC}$ & 2 & $P>L$ & 240 & 88 \\
\hline psaA & exon & 44,617 & 1395 & $G>A$ & $\mathrm{CCC}>\mathrm{CCU}$ & 3 & $P>P$ & 95 & 37 \\
\hline IGR-3 & IGR & 51,329 & - & $C>U$ & - & - & - & 614 & 17 \\
\hline$n d h K$ & exon & 54,554 & 65 & $G>A$ & $U C A>U U A$ & 2 & $S>L$ & 826 & 89 \\
\hline$n d h C$ & exon & 54,719 & 323 & $G>A$ & $A C U>A \cup U$ & 2 & $T>1$ & 816 & 96 \\
\hline IGR-4 & IGR & 59,062 & - & $G>A$ & - & - & - & 349 & 28 \\
\hline$a c c D$ & exon & 62,731 & 815 & $C>U$ & $U C G>U U G$ & 2 & $S>L$ & 65 & 92 \\
\hline$a c c D$ & exon & 63,322 & 1406 & $C>U$ & $C C A>C \cup A$ & 2 & $P>L$ & 302 & 82 \\
\hline psal & exon & 64,351 & 88 & $C>U$ & $C A U>U A U$ & 1 & $H>Y$ & 89 & 84 \\
\hline cemA & exon & 66,260 & 492 & $C>U$ & $U U C>U U U$ & 3 & $F>F$ & 84 & 49 \\
\hline IGR-5 & IGR & 67,928 & - & $C>U$ & - & - & - & 31 & 13 \\
\hline$p s b F$ & exon & 69,182 & 77 & $G>A$ & $U C U>U U U$ & 2 & $S>F$ & 26 & 96 \\
\hline$p s b F$ & $5^{\prime} \cup T R$ & 69,260 & -2 & $G>A$ & - & - & - & 39 & 62 \\
\hline$p s b E$ & exon & 69,306 & 214 & $G>A$ & $C C U>U C U$ & 1 & $P>S$ & 46 & 96 \\
\hline petL & exon & 70,671 & 5 & $C>U$ & $\mathrm{CCU}>\mathrm{CUU}$ & 2 & $P>L$ & 28 & 46 \\
\hline IGR-6 & IGR & 71,784 & - & $C>U$ & - & - & - & 1163 & 99 \\
\hline clpP & exon & 74,986 & 559 & $G>A$ & $C A U>U A U$ & 1 & $H>Y$ & 33 & 97 \\
\hline$p s b N$ & exon & 79,461 & 29 & $G>A$ & $U C U>U U U$ & 2 & $S>F$ & 70 & 30 \\
\hline petB & intron & 80,119 & - & $C>T$ & - & - & - & 56 & 18 \\
\hline petB & exon & 81,188 & 418 & $C>U$ & $C G G>U G G$ & 1 & $R>W$ & 206 & 94 \\
\hline
\end{tabular}


Table 3 RNA editing sites and amino acid changes (Continued)

\begin{tabular}{|c|c|c|c|c|c|c|c|c|c|}
\hline Gene & Subunit & Genome Position & Gene Position & Nucleotide Change & Codon Change & $\begin{array}{l}\text { Edit Position within } \\
\text { Codon }\end{array}$ & $\begin{array}{l}\text { Amino Acid } \\
\text { Change }\end{array}$ & $\begin{array}{l}\text { Sequencing } \\
\text { Depth }\end{array}$ & $\begin{array}{l}\text { Editing } \\
\text { Efficiency }\end{array}$ \\
\hline petB & exon & 81,381 & 611 & $C>U$ & $C C A>C U A$ & 2 & $P>L$ & 207 & 94 \\
\hline rpoA & exon & 83,130 & 830 & $G>A$ & $U C A>U U A$ & 2 & $S>L$ & 144 & 70 \\
\hline rpoA & exon & 83,760 & 200 & $G>A$ & $U C U>U U U$ & 2 & $S>F$ & 76 & 83 \\
\hline rps11 & exon & 84,338 & 108 & $G>A$ & $U U C>\cup U U$ & 3 & $F>F$ & 357 & 55 \\
\hline$r p / 23$ & exon & 91,217 & 89 & $G>A$ & $U C A>U U A$ & 2 & $S>L$ & 25 & 84 \\
\hline rpl23 & exon & 91,235 & 71 & $G>A$ & UCU > UUU & 2 & $S>F$ & 31 & 97 \\
\hline$y c f 2$ & exon & 98,495 & 6863 & $A>G$ & $U A A>U G A$ & 2 & ${ }^{*}>^{*}$ & 6 & 50 \\
\hline$n d h B$ & exon & 99,901 & 1487 & $G>A$ & $C C A>C U A$ & 2 & $P>L$ & 20 & 80 \\
\hline$n d h B$ & exon & 99,955 & 1433 & $G>A$ & $U C A>U U A$ & 2 & $S>L$ & 15 & 40 \\
\hline$n d h B$ & exon & 100,133 & 1255 & $G>A$ & $C A U>U A U$ & 1 & $H>Y$ & 17 & 100 \\
\hline$n d h B$ & exon & 100,276 & 1112 & $G>A$ & $U C A>U U A$ & 2 & $S>L$ & 25 & 88 \\
\hline$n d h B$ & exon & 100,558 & 830 & $G>A$ & $U C A>U U A$ & 2 & $S>L$ & 10 & 70 \\
\hline$n d h B$ & exon & 101,328 & 746 & $G>A$ & $U C U>U U U$ & 2 & $S>F$ & 9 & 22 \\
\hline$n d h B$ & exon & 101,337 & 737 & $G>A$ & $C C A>C U A$ & 2 & $P>L$ & 11 & 64 \\
\hline$n d h B$ & exon & 101,488 & 586 & $G>A$ & $C A U>\cup A U$ & 1 & $H>Y$ & 11 & 82 \\
\hline$n d h B$ & exon & 101,607 & 467 & $G>A$ & $C C A>C U A$ & 2 & $P>L$ & 23 & 91 \\
\hline$n d h B$ & exon & 101,925 & 149 & $G>A$ & $U C A>U \cup A$ & 2 & $S>L$ & 23 & 91 \\
\hline rps 12 & intron & 103,149 & - & $G>A$ & - & - & - & 87 & 67 \\
\hline rps 12 & intron & 103,290 & - & $G>A$ & - & - & - & 115 & 85 \\
\hline IGR-7 & IGR & 104,462 & - & $G>A$ & - & - & - & 629 & 30 \\
\hline rrn16s & exon & 106,489 & 577 & $G>A / U / C$ & - & - & - & 1404 & 49 \\
\hline rrn16s & exon & 107,021 & 45 & $U>A / G$ & - & - & - & 1112 & 41 \\
\hline$r r n 23 S$ & exon & 111,418 & 881 & $U>A / G / C$ & - & - & - & 3163 & 73 \\
\hline$n d h F$ & exon & 116,076 & 1734 & $C>U$ & $A \cup G>A \cup A$ & 3 & $M>1$ & 23 & 26 \\
\hline$n d h F$ & exon & 117,520 & 290 & $G>A$ & $U C A>\cup \cup A$ & 2 & $S>L$ & 21 & 38 \\
\hline$n d h D$ & exon & 121,618 & 1298 & $G>A$ & $U C A>U U A$ & 2 & $S>L$ & 52 & 92 \\
\hline$n d h D$ & exon & 122,029 & 887 & $G>A$ & $\mathrm{CCC}>\mathrm{CUC}$ & 2 & $P>L$ & 12 & 58 \\
\hline$n d h D$ & exon & 122,242 & 674 & $G>A$ & $U C A>U U A$ & 2 & $S>L$ & 40 & 85 \\
\hline$n d h D$ & exon & 122,317 & 599 & $G>A$ & $U C A>\cup \cup A$ & 2 & $S>L$ & 46 & 76 \\
\hline$n d h D$ & exon & 122,533 & 383 & $G>A$ & $U C A>U U A$ & 2 & $S>L$ & 28 & 75 \\
\hline$n d h D$ & exon & 122,914 & 2 & $G>A$ & $A C G>A \cup G$ & 2 & $\mathrm{~T}>\mathrm{M}$ & 33 & 64 \\
\hline$n d h E$ & exon & 123,626 & 233 & $G>A$ & $C C A>C U A$ & 2 & $P>L$ & 110 & 9 \\
\hline$n d h A$ & exon & 125,765 & 961 & $G>A$ & $C C U>U C U$ & 1 & $P>S$ & 334 & 94 \\
\hline$n d h A$ & exon & 127,589 & 341 & $G>A$ & $U C A>U U A$ & 2 & $S>L$ & 99 & 79 \\
\hline$n d h H$ & exon & 128,810 & 303 & $G>A$ & $A \cup C>A \cup U$ & 3 & $1>1$ & 44 & 25 \\
\hline$y c f 1$ & exon & 131,464 & 4236 & $G>A$ & $\mathrm{CGC}>\mathrm{CGU}$ & 3 & $R>R$ & 160 & 19 \\
\hline$y c f 1$ & exon & 133,945 & 1755 & $G>A$ & $U U C>U U U$ & 3 & $F>F$ & 250 & 17 \\
\hline
\end{tabular}

Editing efficiency is counted by edited reads divided by total mapped reads at the same site

If the sequencing depth of an editing site is less than 30 , the edit rate may have a great error

The editing sites in the IR region are calculated only once

IGR Intergenic region

UTR untranslated region, it belongs to exon

*: Stop codon 


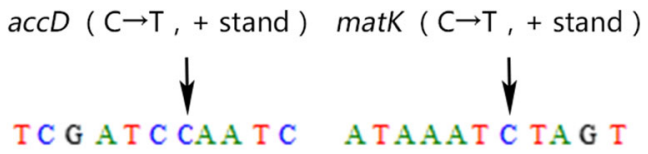

gDNA
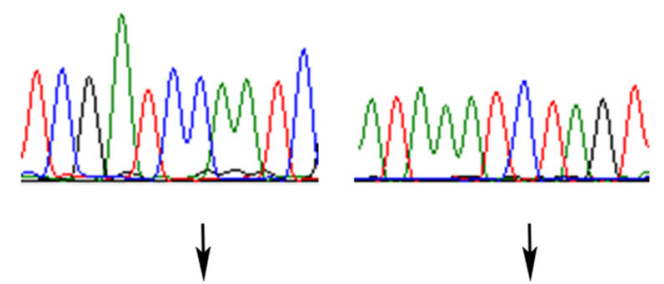

I C G A T C TAA T C

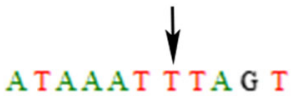

CDNA
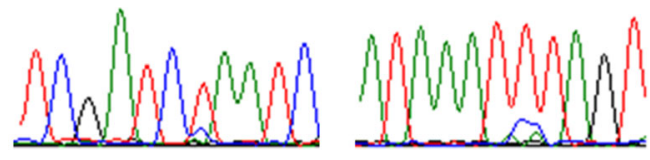

Fig. 5 Validation of inferred editing sites from RNA-Seq by Sanger sequencing. Sequencing chromatogram traces from two exemplary gene loci, accD and matK, are shown. The editing positions are highlighted by arrows. The top trace is genomic DNA (gDNA), and the bottom trace is complementary DNA (CDNA)

\section{Discussion}

The study presented here reported a complete cp genome sequence of $B$. platyphylla. In general, it had typical characteristics of angiosperm cp genomes and had little difference between closely related species. However, some of the phenomena attracted our attention.

Chloroplast microsatellites are some of the most common cp molecular markers. They are often used to investigate the evolution and systematics of genera and higher taxonomic ranks [32-36]. Although SSRs have now almost become the standard analysis in cp genome research, their scope of application is still open to question. Bang et al. have warned that attention must be paid to the risk of using amplicon-sized cPSSR markers for genetic relationship studies [37]. In our study, we found that there were more than $100 \mathrm{cpSSR}$ loci in the $B$. platyphylla cp genome, but only one possible cpSSR locus among the three Betula species was predicted by CandiSSR. We also further identified the candidate polymorphic cPSSR loci among four typical species of Fagales (B. platyphylla, C. mollissima, J. regia and $M$. rubra) and obtained three possible loci. This seems to indicate that the closer the relationship between the species, the fewer candidate cPSSR loci were found for differentiating the species. For species that are closely related, a small number of cpSSRs may not be able to distinguish them accurately, especially depending on

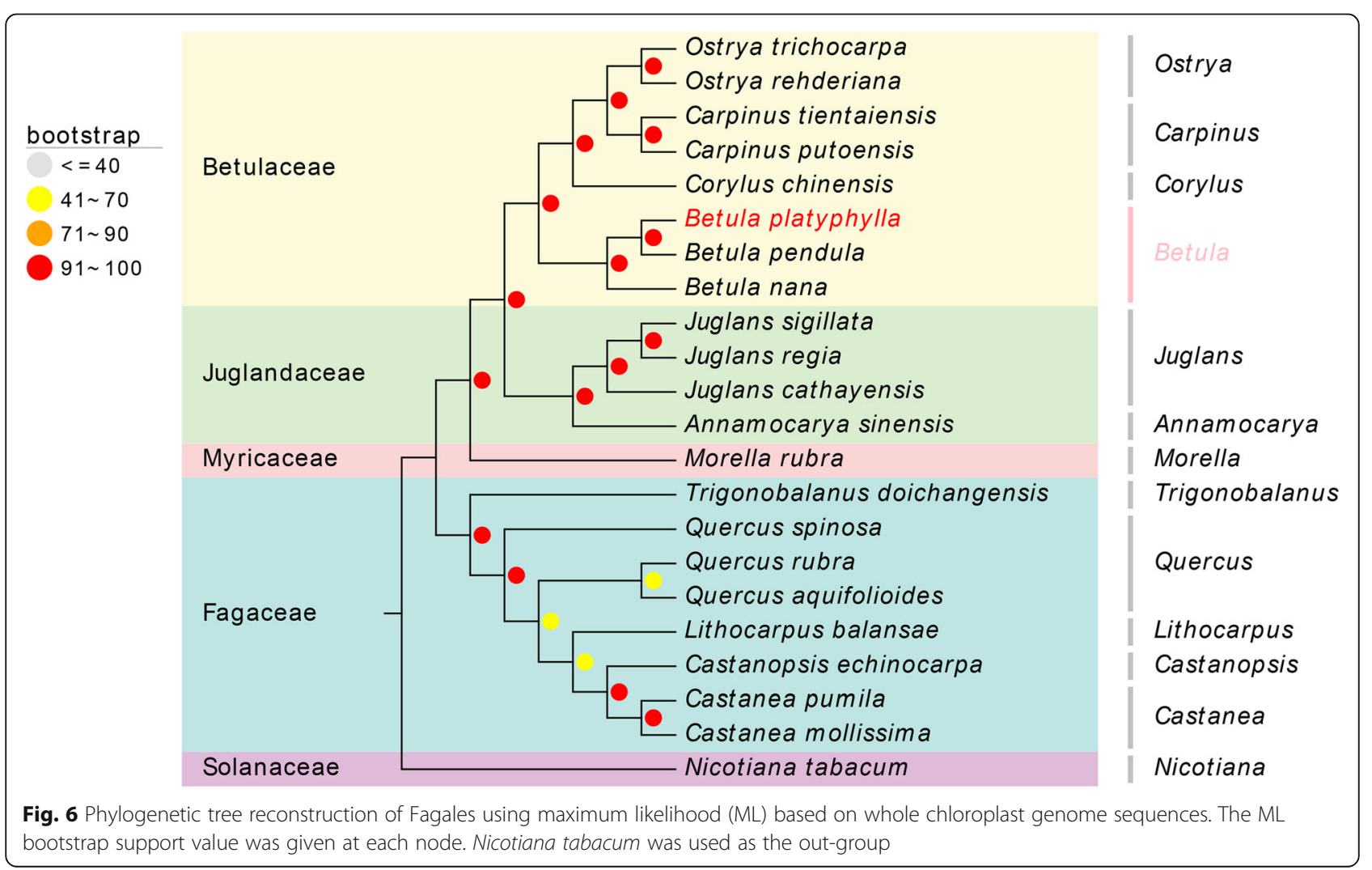


length alone. Therefore, we sometimes need more information, such as SNPs or INDEL, to differentiate closely related species.

We also reconstructed the phylogenetic tree of Fagales. Our ML tree formed four major clades and was highly consistent with previous research [38, 39]. Among the three closely related Betula species, B. nana is significantly different from the other two species, not only in morphology but also in the growth environment. The Betula branch had a strongly supported topology and showed that B. platyphylla and B. pendula have a closer evolutionary relationship. A previously reconstructed phylogeny for Betula species that is based on whole-genome SNP information reached the same conclusion [40].

RNA editing in the B. platyphylla cp genome is also one of the concerns of our study. As an important type of posttranscriptional regulation, it was previously widely believed that there were 30 50 RNA editing sites in a large majority of cp genomes [41-44]. The substitution is always either from cytidine $(\mathrm{C})$ to uridine $(\mathrm{U})$ or, less commonly, uridine $(\mathrm{U})$ to cytidine $(\mathrm{C})[45,46]$. In the past, software was used to predict RNA editing sites, but the software accuracy was generally low, and it could not predict synonymous mutation sites. In recent years, the advent of NGS improved the sensitivity of RNA editing site identification. An increasing number of editing sites that are not only in coding regions but also in noncoding and intergenic regions have been identified [47-49]. To reduce the false-positive, at most, one mismatch was allowed in an aligned reads and no gap open or gap extension was allowed when mapping to the reference genome. This inevitably lost some information and might miss possible editing sites that are very close to each other. However, it clearly improved the accuracy of the recognition. Finally, we identified 80 editing sites in the whole cp genome, including coding regions, noncoding regions and intergenic regions. Most of the substitutions were $\mathrm{C}$ to $\mathrm{U}$, but a small number of other types that have never been reported in flowering plants were also detected. Except for the rRNAs, the editing sites that are not $\mathrm{C}$ to $\mathrm{U}$ are either low in sequencing depth or low in editing efficiency. This means that these edited transcripts are lower in expression, which seems to explain why these types of substitutions have not previously been discovered. Although we did not find any editing sites in B. platyphylla tRNA, some specific editing sites were identified in rRNAs. Unlike ordinary RNA editing, the editing sites in B. platyphylla rRNA can be replaced with different kinds of bases. Until now, the phenomenon of rRNA editing has been found only in algae chloroplasts [50-52]. Although other B. platyphylla RNA-Seq data also led to the same conclusion, we still doubted whether these editing sites were species specific. Excitingly, from public RNA-Seq data for
Arabidopsis thaliana (https://www.ncbi.nlm.nih.gov/sra/, SRR682085, SRR6852086), we found almost the same editing modes at the same loci in the cp rRNA of $A$. thaliana. This indicates that cp rRNA editing may be somewhat conserved across species. It should be noted that most of the rRNAs were removed in the process of library construction; in fact, the expression of cp rRNA is far higher than the value we detected. Perhaps there is some mechanism to promote the conversion from one base to several other bases in a huge number of transcripts. In recent years, great progress has been made in the molecular mechanism of RNA editing, especially for $\mathrm{C}$-to- $\mathrm{U}$ conversion. Some pentatricopeptide repeat (PPR) proteins and other factors, such as the proteins CRR, RIP/MORF, ORRM and OZ, have been found to form an RNA editosome and to guide the hydrolytic deamination of a cytosine to a uracil base [53-57]. Obviously, these studies are insufficient to elucidate the essence of the problem, especially for those other than for C-to-U editing. For a long time, RNA editing was believed to be a mechanism at the posttranscriptional level, probably acting as buffer to less favored mutations in the genomic coding sequences [56]. However, with the discovery of more editing sites, RNA editing seems to have some other effects. Notably, we found that, other than for the stop codon conversion, the 6 amino acid synonymous conversions increased the RSCU value which suggests a plant codon optimization phenomenon to improve protein expression. Moreover, there is no theoretical explanation for the cp rRNA editing phenomenon. Because rRNA is the main component of the ribosome and catalyzes peptide bond formation, these editing sites may change the ribosome structure or influence protein synthesis. Although sometimes RNA editing is important, is it necessary for all of these editing sites? Especially for those sites with low expression or low editing efficiency, we suspected that some of them may be the results of accidental effects of editosomes and transcripts. Of course, all of these conjectures require further confirmation through in vitro and in vivo studies.

The cp genome has obvious prokaryotic characteristics in codon selection, especially in initiation codon selection [58]. Many kinds of initiation codons, including ACG, GTG and ATA (but not ATG), have been found in chloroplasts, which is conserved across closely related species [59]. Although the sequence logo clearly shows the trend of initiation codon selection in different species, most of the current cp genome annotations were based on software prediction, which may be different from the real situation. The use of alternative initiation codons leads to two choices in the stages of translation and transcription: one is to use the Non-ATG as the start codon directly, and the other is to edit it back to ATG by RNA editing. In the B. platyphylla $\mathrm{cp}$, the initiation codons of the $n d h D$ transcripts were obviously 
edited, but those of rps19 and $p s b C$ were not. The Shine-Dalgarno Sequence seems to facilitate translation initiation from the GUG [58, 60]. Recent studies have also surprised us with the translation of some unedited transcripts, which raises the possibility that ACG in $n d h D$ can be utilized as an initiator codon in chloroplasts [61, 62]. Furthermore, attention should be given to whether unedited transcripts have some functions.

\section{Conclusions}

In conclusion, we sequenced and investigated the complete $\mathrm{cp}$ genome sequence of $B$. platyphylla. The $\mathrm{cp}$ genome of B. platyphylla has a typical land plant cp genome structure and is highly similar to other $\mathrm{cp}$ genome sequences of Fagales. There were 3 genes using alternative initiation codons. More RNA editing sites were detected than ever before, and some had never been reported. This also helped us to determine the phylogenetic relationships among some species of Fagales. Our research will facilitate genomic, genetic engineering and phylogenetic studies of this important species. In the future, we will focus on molecular mechanisms that are involved in transcriptional regulation and translational modification of the cp by using new technologies and methods. We hope that these studies will help develop new varieties with higher photosynthetic efficiency.

\section{Additional file}

Additional file 1: Table S1. Taxa and ID of the selected species. Table S2. The related information of primers. Table S3. Taxa and ID of the selected Fagales. Table S4. Codon usage frequency and RSCU value of the B. platyphylla chloroplast genome. Table S5. Simple sequence repeats within the Betula platyphylla chloroplast genome. Table $\mathbf{5 6 .}$ Candidate polymorphic SSRs and primers. Figure S1. Amino acid composition of protein-coding gene in the B. platyphylla chloroplast genome. Figure S2. Genome coverage distribution curve of RNASeq.Window length: $100 \mathrm{nt}$; step size: $50 \mathrm{nt}$. Figure S3. Gene body coverage distribution curve of RNA-Seq.All genes and sequencing depth have been normalized. Figure S4. Number of classified SSR repeat types (considering sequence complementary). (DOCX $2105 \mathrm{~kb}$ )

\section{Abbreviations}

APG: Angiosperm Phylogeny Group; CNS: Conserved non-coding sequences; cp: Chloroplast; CTAB: Cetyl trimethylammonium bromide; IGR: Intergenic region; IR: Inverted repeat; LSC: Large single copy; ML: Maximum likelihood; NCBI: National Center for Biotechnology Information; NGS: Next-generationsequencing; PPR: Pentatricopeptide repeat; RSCU: Relative synonymous codon usage; SNP: Single-nucleotide polymorphism; SSC: Small single copy; SSR: Simple sequence repeat; UTR: Untranslated region

\section{Acknowledgements}

We sincerely thank Yi Liu, Caixiao Liu and Xiaosha Sun for their help with the DNA and RNA extraction.

\section{Funding}

This research was supported by the National Natural Science Foundation of China (No. 31770712) and the Fundamental Research Funds for the Central Universities (2572015EA03). The funding bodies had no role in the design of the study, collection, analysis, or interpretation of data or in the writing of the manuscript.

\section{Availability of data and materials}

The data sets supporting the results of this article are included within the manuscript and its additional files. RNA-Seq data of B. platyphylla leaves were submitted to the NCBI Sequence Read Archive (SRA) under accession numbers SRR7051061, SRR7051062, SRR7051063. The complete cp genome of B. platyphylla was submitted to GenBank under the accession number $\mathrm{MH} 205735$. All the $\mathrm{cp}$ genome information was also stored at http://www.wangsui.net.cn/resource/database/public/plant/Betula_platyphylla/chloroplast/.

Authors' contributions

SW assembled, annotated and analysed the $\mathrm{CP}$ genome sequence of $B$. platyphylla. SW wrote the manuscript. CPY, XYZ, SC and GZQ revised the manuscript. All authors approved the final manuscript.

\section{Ethics approval and consent to participate}

The Betula platyphylla materials used in the study were preserved in the State Key Laboratory of Tree Genetics and Breeding and were planted on the campus of Northeast Forestry University (latitude $45^{\circ} 42^{\prime} 57.58^{\prime \prime} \mathrm{N}$, longitude $\left.126^{\circ} 37^{\prime} 44.41^{\prime \prime} \mathrm{E}\right)$. As researches of the laboratory, we are allowed to use these materials for research. Samplings of these materials were performed in compliance with institutional, national, and international guidelines.

Consent for publication

Not applicable.

\section{Competing interests}

The authors declare that they have no competing interests.

\section{Publisher's Note}

Springer Nature remains neutral with regard to jurisdictional claims in published maps and institutional affiliations.

Received: 4 May 2018 Accepted: 30 November 2018

Published online: 20 December 2018

\section{References}

1. Zhang Z. Dendrology. 2nd ed. Beijing: China Forestry Publishing House; 2008

2. Mobile Reference. The Illustrated Encyclopedia of Trees and Shrubs: An Essential Guide to Trees and Shrubs of the World. Boston: Mobile Reference; 2008.

3. Wang Y, Gao C, Zheng L, Liu G, Jiang J, Yang C. Building an mRNA transcriptome from the shoots of Betula platyphylla by using Solexa technology. Tree Genet Genomes. 2012;8(5):1031-40.

4. Wang S, Zhao H, Jiang J, Liu G, Yang C. Analysis of three types of triterpenoids in tetraploid white birches (Betula platyphylla Suk.) and selection of plus trees. J Forestry Res. 2015;26(3):623-33.

5. Krasutsky PA. Birch bark research and development. Nat Prod Rep. 2006; 23(6):919-42.

6. Gray MW. The evolutionary origins of plant organelles. In: Daniell H, Chase C, editors. Molecular biology and biotechnology of plant organelles: chloroplasts and mitochondria. Dordrecht: Springer Netherlands; 2004. p. 15-36.

7. McFadden Gl. Chloroplast origin and integration. Plant Physiol. 2001;125(1): 50-3

8. Ohyama K, Fukuzawa H, Kohchi T, Shirai H, Sano T, Sano S, Umesono K, Shiki Y, Takeuchi M, Chang Z, et al. Chloroplast gene organization deduced from complete sequence of liverwort Marchantia polymorpha chloroplast DNA. NATURE. 1986;322(6079):572-4.

9. Shinozaki K, Ohme M, Tanaka M, Wakasugi T, Hayashida N, Matsubayashi T, Zaita N, Chunwongse J, Obokata J, Yamaguchi-Shinozaki K, et al. The complete nucleotide sequence of the tobacco chloroplast genome: its gene organization and expression. EMBO J. 1986;5(9):2043-9.

10. Moore MJ, Dhingra A, Soltis PS, Shaw R, Farmerie WG. Rapid and accurate pyrosequencing of angiosperm plastid genomes. BMC Plant Biol. 2006;6(1):17.

11. Stadermann KB, Weisshaar B, Holtgräwe D. SMRT sequencing only de novo assembly of the sugar beet (Beta vulgaris) chloroplast genome. BMC Bioinformatics. 2015;16(1):295.

12. Ferrarini $M$, Moretto $M$, Ward JA, Surbanovski $N$, Stevanovic V, Giongo L Viola R, Cavalieri D, Velasco R, Cestaro A, et al. An evaluation of the PacBio 
RS platform for sequencing and de novo assembly of a chloroplast genome. BMC Genomics. 2013;14:670.

13. Soorni A, Haak D, Zaitlin D, Bombarely A. Organelle_PBA, a pipeline for assembling chloroplast and mitochondrial genomes from PacBio DNA sequencing data. BMC Genomics. 2017;18(1):49.

14. Lin CP, Huang JP, Wu CS, Hsu CY, Chaw SM. Comparative chloroplast genomics reveals the evolution of Pinaceae genera and subfamilies. Genome Biol Evol. 2010;2:504-17.

15. Chumley TW, Palmer JD, Mower JP, Fourcade HM, Calie PJ, Boore JL, Jansen RK. The complete chloroplast genome sequence of Pelargonium $x$ hortorum: organization and evolution of the largest and most highly rearranged chloroplast genome of land plants. Mol Biol Evol. 2006;23(11):2175-90

16. Daniell H, Lin C, Yu M, Chang W. Chloroplast genomes: diversity, evolution, and applications in genetic engineering. Genome Biol. 2016;17(1):134.

17. Chateigner-Boutin AL, Small I. Plant RNA editing. RNA Biol. 2010;7(2):213-9.

18. Ravi V, Khurana JP, Tyagi AK, Khurana P. An update on chloroplast genomes. Plant Syst Evol. 2008;271(1-2):101-22.

19. Porebski S, Bailey LG, Baum BR. Modification of a CTAB DNA extraction protocol for plants containing high polysaccharide and polyphenol components. Plant Mol Biol REP. 1997;15(1):8-15.

20. Patel RK, Jain M. NGS QC Toolkit: A Toolkit for quality control of next generation sequencing data. PLoS One. 2012;7(2):e30619.

21. Li H: Aligning sequence reads, clone sequences and assembly contigs with BWA-MEM. arXiv preprint arXiv:1303.3997 2013.

22. Hernandez D, Francois $P$, Farinelli L, Osteras M, Schrenzel J. De novo bacterial genome sequencing: millions of very short reads assembled on a desktop computer. Genome Res. 2008;18(5):802-9.

23. Boetzer M, Henkel CV, Jansen HJ, Butler D, Pirovano W. Scaffolding preassembled contigs using SSPACE. Bioinformatics. 2011;27(4):578-9.

24. Liu C, Shi L, Zhu Y, Chen H, Zhang J, Lin X, Guan X. CpGAVAS, an integrated web server for the annotation, visualization, analysis, and GenBank submission of completely sequenced chloroplast genome sequences. BMC Genomics. 2012;13:715

25. Lowe TM, Eddy SR. tRNAscan-SE: a program for improved detection of transfer RNA genes in genomic sequence. Nucleic Acids Res. 1997;25(5):955-64.

26. Lohse M, Drechsel O, Kahlau S, Bock R. OrganellarGenomeDRAW--a suite of tools for generating physical maps of plastid and mitochondrial genomes and visualizing expression data sets. Nucleic Acids Res. 2013; 41 (W1):W575-81.

27. Xia E, Yao Q, Zhang H, Jiang J, Zhang L, Gao L. CandisSR: an efficient pipeline used for identifying candidate polymorphic SSRs based on multiple assembled sequences. Front Plant Sci. 2016;6:1171.

28. Castandet B, Hotto AM, Strickler SR, Stern DB. ChloroSeq, an optimized chloroplast RNA-Seq Bioinformatic pipeline, reveals remodeling of the Organellar transcriptome under heat stress. G3: Genes, Genomes, Genetics. 2016;6(9):2817-27.

29. Liu Q, Xue Q. Comparative studies on codon usage pattern of chloroplasts and their host nuclear genes in four plant species. J Genet. 2005;84(1):55-62.

30. Shapiro JA, von Sternberg $R$. Why repetitive DNA is essential to genome function. Biol Rev Camb Philos Soc. 2005;80(2):227-50.

31. Cournac A, Koszul R, Mozziconacci J. The 3D folding of metazoan genomes correlates with the association of similar repetitive elements. Nucleic Acids Res. 2016:44(1):245-55.

32. Provan J, Corbett G, McNicol JW, Powell W. Chloroplast DNA variability in wild and cultivated rice (Oryza spp.) revealed by polymorphic chloroplast simple sequence repeats. GENOME. 1997;40(1):104-10.

33. Angioi SA, Desiderio F, Rau D, Bitocchi E, Attene G, Papa R. Development and use of chloroplast microsatellites in Phaseolus spp. and other legumes. Plant Biol. 2009;11(4):598-612

34. Ebert $D$, Peakall R. A new set of universal de novo sequencing primers for extensive coverage of noncoding chloroplast DNA: new opportunities for phylogenetic studies and cpSSR discovery. Mol Ecol Resour. 2009;9(3):777-83.

35. Wheeler GL, Dorman HE, Buchanan A, Challagundla L, Wallace LE. A review of the prevalence, utility, and caveats of using chloroplast simple sequence repeats for studies of plant biology. Appl Plant Sci. 2014;2(12):1400059.

36. Cheng Y, de Vicente MC, Meng H, Guo W, Tao N, Deng X. A set of primers for analyzing chloroplast DNA diversity in Citrus and related genera. Tree Physiol. 2005;25(6):661-72.

37. Bang SW, Chung S. One size does not fit all: the risk of using amplicon size of chloroplast SSR marker for genetic relationship studies. Plant Cell Rep. 2015;34(10):1681-3.
38. Larson-Johnson K. Phylogenetic investigation of the complex evolutionary history of dispersal mode and diversification rates across living and fossil Fagales. New Phytol. 2016;209(1):418-35.

39. Liu L, Li R, Worth JRP, Li X, Li P, Cameron KM, Fu C. The complete chloroplast genome of Chinese bayberry (Morella rubra, Myricaceae): implications for understanding the evolution of Fagales. Front Plant Sci. 2017;8:968.

40. Salojärvi J, Smolander O, Nieminen K, Rajaraman S, Safronov O, Safdari P, Lamminmäki A, Immanen J, Lan T, Tanskanen J, et al. Genome sequencing and population genomic analyses provide insights into the adaptive landscape of silver birch. Nat Genet. 2017;49(6):904.

41. Wang W, Zhang W, Wu Y, Maliga P, Messing J. RNA editing in chloroplasts of Spirodela polyrhiza, an Aquatic Monocotelydonous Species. Plos One. 2015;10(10):e140285.

42. He P, Huang S, Xiao G, Zhang Y, Yu J. Abundant RNA editing sites of chloroplast protein-coding genes in Ginkgo biloba and an evolutionary pattern analysis. BMC Plant Biol. 2016;16(1):257.

43. Jiang Y, Fan SL, Song MZ, Yu JN, Yu SX. Identification of RNA editing sites in cotton (Gossypium hirsutum) chloroplasts and editing events that affect secondary and three-dimensional protein structures. Genet Mol Res. 2012; 11(2):987-1001.

44. Kugita M. RNA editing in hornwort chloroplasts makes more than half the genes functional. Nucleic Acids Res. 2003;31(9):2417-23.

45. Knie N, Grewe F, Fischer S, Knoop V. Reverse U-to-C editing exceeds C-to-U RNA editing in some ferns - a monilophyte-wide comparison of chloroplast and mitochondrial RNA editing suggests independent evolution of the two processes in both organelles. BMC Evol Biol. 2016;16(1):134.

46. Tillich M, Lehwark P, Morton BR, Maier UG. The evolution of chloroplast RNA editing. Mol Biol Evol. 2006;23(10):1912-21.

47. Chen T, Liu Y, Wang X, Wu C, Huang C, Chang C. Whole plastid transcriptomes reveal abundant RNA editing sites and differential editing status in Phalaenopsis aphrodite subsp. formosana. Bot Stud. 2017:58(1):38.

48. Wang M, Liu H, Ge L, Xing G, Wang M, Weining S, Nie X. Identification and analysis of RNA editing sites in the chloroplast transcripts of Aegilops tauschii L. Genes-Basel. 2017;8(1):13.

49. Bentolila S, Oh J, Hanson MR, Bukowski R. Comprehensive high-resolution analysis of the role of an Arabidopsis gene family in RNA editing. PLoS Genet. 2013;9(6):e1003584.

50. Shi C, Wang S, Xia E, Jiang J, Zeng F, Gao L. Full transcription of the chloroplast genome in photosynthetic eukaryotes. Sci Rep-UK. 2016;6(1):30135.

51. Dang Y, Green BR. Substitutional editing of Heterocapsa triquetra chloroplast transcripts and a folding model for its divergent chloroplast 165 rRNA. Gene. 2009;442(1-2):73-80.

52. Wang $Y$, Morse $D$. Rampant polyuridylylation of plastid gene transcripts in the dinoflagellate Lingulodinium. Nucleic Acids Res. 2006;34(2):613-9.

53. Bentolila S, Heller WP, Sun T, Babina AM, Friso G, van Wijk KJ, Hanson MR. RIP1, a member of an Arabidopsis protein family, interacts with the protein RARE1 and broadly affects RNA editing. Proc Natl Acad Sci. 2012;109(22): E1453-61.

54. Takenaka M, Zehrmann A, Verbitskiy D, Kugelmann M, Hartel B, Brennicke A. Multiple organellar RNA editing factor (MORF) family proteins are required for RNA editing in mitochondria and plastids of plants. Proc Natl Acad Sci. 2012;109(13):5104-9.

55. Sun T, Shi X, Friso G, Van Wijk K, Bentolila S, Hanson MR, Zinc Finger A, Motif-Containing Protein I. Essential for chloroplast RNA editing. PLoS Genet. 2015;11(3):e1005028.

56. Ichinose M, Sugita M. RNA editing and its molecular mechanism in plant organelles. Genes-Basel. 2017:8(1):5.

57. Sun T, Germain A, Giloteaux L, Hammani K, Barkan A, Hanson MR, Bentolila S. An RNA recognition motif-containing protein is required for plastid RNA editing in Arabidopsis and maize. Proc Natl Acad Sci. 2013;110(12):E1169-78.

58. Scharff $L B$, Ehrnthaler $M$, Janowski $M$, Childs LH, Hasse C, Gremmels J, Ruf $\mathrm{S}$, Zoschke R, Bock R. Shine-Dalgarno sequences play an essential role in the translation of plastid mRNAs in tobacco. Plant Cell. 2017; 29(12):3085-101.

59. Wu Z, Gui S, Quan Z, Pan L, Wang S, Ke W, Liang D, Ding Y. A precise chloroplast genome of Nelumbo nucifera (Nelumbonaceae) evaluated with sanger, Illumina MiSeq, and PacBio RS II sequencing platforms: insight into the plastid evolution of basal eudicots. BMC Plant Biol. 2014;14:289.

60. Kuroda H, Suzuki H, Kusumegi T, Hirose T, Yukawa Y, Sugiura M. Translation of psbC mRNAs starts from the downstream GUG, not the upstream AUG, 
and requires the extended Shine-Dalgarno sequence in tobacco chloroplasts. Plant Cell Physiol. 2007;48(9):1374-8.

61. Zandueta-Criado A, Bock R. Surprising features of plastid ndhD transcripts: addition of non-encoded nucleotides and polysome association of mRNAs with an unedited start codon. Nucleic Acids Res. 2004;32(2):542-50.

62. Zoschke R, Bock R. Chloroplast translation: structural and functional organization, operational control, and Regulation. Plant Cell. 2018;30(4): 745-70.

Ready to submit your research? Choose BMC and benefit from:

- fast, convenient online submission

- thorough peer review by experienced researchers in your field

- rapid publication on acceptance

- support for research data, including large and complex data types

- gold Open Access which fosters wider collaboration and increased citations

- maximum visibility for your research: over $100 \mathrm{M}$ website views per year

At $\mathrm{BMC}$, research is always in progress.

Learn more biomedcentral.com/submissions 